\title{
Analysis of the expression of plasma omentin-1 level in colorectal cancer and its correlation with prognosis
}

\author{
Zhangdong Feng ${ }^{1}$, Haitao Sun ${ }^{1}$, Peng Liu ${ }^{1}$, Wei Shi ${ }^{1}$, Wei Han ${ }^{1}$, Lifeng $\mathrm{Ma}^{2}$ \\ ${ }^{1}$ Department of General Surgery, Beijing Luhe Hospital, Affiliated to Capital Medical University, Beijing, China; ${ }^{2}$ General Surgery (Minimally \\ Invasive Biliary Surgery), The Second Hospital of Hebei Medical University, Shijiazhuang, China \\ Contributions: (I) Conception and design: Z Feng, W Han, L Ma; (II) Administrative support: H Sun; (III) Provision of study materials or patients: Z \\ Feng, H Sun, P Liu, W Shi; (IV) Collection and assembly of data: All authors; (V) Data analysis and interpretation: Z Feng, P Liu, W Shi, W Han, L \\ Ma; (VI) Manuscript writing: All authors; (VII) Final approval of manuscript: All authors. \\ Correspondence to: Wei Han. Department of General Surgery, Beijing Luhe Hospital, Affiliated to Capital Medical University, No.82 Xinhua South \\ Street, Tongzhou District, Beijing 101100, China. Email: fzd1866@163.com; Lifeng Ma. No. 215, Heping West Road, Shijiazhuang 050000, China. \\ Email: season0325@126.com.
}

Background: Colorectal cancer (CRC) is a malignant tumor of the digestive tract, with high incidence and mortality. This study aims to investigate the expression of plasma omentin-1 level in patients with CRC and its correlation with the prognosis of patients.

Methods: Clinical data of 319 patients (case group) who underwent radical resection of CRC in our hospital from June 2015 to March 2018 were collected, simultaneously, 300 people without the apparent physical disease who underwent physical examination in our hospital during the same period were randomly selected as the control group, plasma omentin-1 levels in the two groups were compared by T-test. Cancer patients were followed up, and multi-factor COX regression analysis was used to analyze the factors affecting patients' recurrence and survival.

Results: The level of plasma omentin-1 in patients with colorectal cancer is closely related to TNM staging and lymph node metastasis. The CRC case group was significantly reduced after surgery, but it was still higher than that in the control group $(\mathrm{P}<0.05)$. At the end of the follow-up, 28 cases were lost to followup, and 291 cases $(91.22 \%)$ were effectively followed up. During the follow-up period, the recurrence and metastasis rate was $30.58 \%(89 / 291)$, and the mortality rate was $19.26 \%(56 / 291)$. According to the multifactor analysis, the risk factors for the recurrence of CRC patients were TNM stage III-IV (OR $=1.978,95 \%$ CI: 1.088-3.596), lymph node metastasis (OR $=2.073,95 \% \mathrm{CI}: 1.179-3.645)$ and omentin-1 level $\geq 50 \mathrm{ng} / \mathrm{mL}$ $(\mathrm{OR}=3.347,95 \% \mathrm{CI}: 1.416-7.912)$. The risk factors for the survival of patients with CRC were poorly differentiated (OR =1.960, 95\% CI: 0.985-3.900), lymph node metastasis (OR =2.452, 95\% CI: 1.213-4.956), vascular carcinoma thrombus $(\mathrm{OR}=2.026,95 \% \mathrm{CI}: 1.036-3.960)$ and omentin-1 level $\geq 50 \mathrm{ng} / \mathrm{mL}(\mathrm{OR}$ $=2.067,95 \%$ CI: $1.013-4.218)$.

Conclusions: The level of plasma omentin-1 is highly expressed in patients with CRC, an independent risk factor for the recurrence and survival of patients.

Keywords: Omentin-1; colorectal cancer (CRC); prognosis; recurrence; survival

Submitted Aug 04, 2020. Accepted for publication Sep 25, 2020.

doi: $10.21037 /$ tcr-20-2836

View this article at: http://dx.doi.org/10.21037/tcr-20-2836 


\section{Introduction}

Colorectal cancer (CRC) is a common malignant tumor of the digestive tract clinically, which ranks third and fifth of the incidence and mortality of all tumors $(1,2)$. The main clinical treatment for patients with colorectal cancer is surgical resection, which has a significant short-term effect, but the higher recurrence and metastasis rate in the later stage still seriously threatens the safety of patients (3). How to find relapsed patients as soon as possible and early intervention treatment are the main means to prolong their lives, and the research on the risk factors that affect the prognosis of colorectal cancer patients mostly focuses on macroscopic pathological parameters and common tumor markers $(1,3,4)$. As a loose connective tissue composed of fat cells, adipose tissue is also an essential endocrine organ of the human body, which can mainly secrete adipokines, including adiponectin, omentin-1, leptin (5). Studies have shown that adipokines have a specific relationship with pancreatic cancer (6), ovarian cancer (7), and other cancers. And omentin-1 can affect the proliferation and apoptosis of colorectal cancer stem cells through the PI3K/Akt signaling pathway (8), The abnormal activation of these regulatory adipokines and their receptors also plays a vital role in the occurrence and progression of CRC (9). However, the relationship of omentin-1 with the prognosis of patients with CRC is not clear. In this study, a prospective follow-up study was conducted to explore the expression of adipokinin omentin-1 in patients with CRC and its correlation with the prognosis of patients. We present the following article in accordance with the REMARK reporting checklist (available at http://dx.doi.org/10.21037/tcr-20-2836).

\section{Methods}

\section{Subjects}

Three hundred nineteen patients who underwent radical resection of CRC in our hospital from June 2015 to March 2018 were enrolled as the case group, including 204 cases of colon cancer and 115 cases of rectal cancer. The ratio of male to female was $182: 137$, with an average age of $(58.65 \pm 12.85)$ years old. There were 104 patients with lymph node metastasis. TNM staging situation was: 21 cases in stage I, 114 cases in stage II, 136 cases in stage III, 48 cases in stage IV. Simultaneously, we randomly selected 300 healthy people who had no apparent physiological diseases by health examinations in our hospital during the same period and agreed to take part in this investigation and study as a control group. In this group, the male to female ratio was $178: 128$, with an average age of $(57.42 \pm 13.04)$ years old. There is no statistically significant difference between the two groups $(\mathrm{P}>0.05)$. This study was approved by Beijing Luhe Hospital, Affiliated to Capital Medical University (No. 2020-LHKY-033-01). The study was conducted in accordance with the Declaration of Helsinki (as revised in 2013). Written informed consent was obtained from the patient.

\section{Inclusion and exclusion criteria}

The inclusion criteria for the study were (I) patients with CRC diagnosed by pathological examination. (II) Not receiving radiotherapy and chemotherapy before the operation. (III) No history of the malignant tumor. (IV) Complete clinical data. (V) Given informed consent to this study. The exclusion criteria for the study were (I) with intestinal inflammation or familial polyposis. (II) Distant metastasis was found before the operation. (III) Other diseases accompanied the patient, including severe liver and kidney dysfunction, cardiac insufficiency. (IV) There was a recurrence within one month after surgery or death.

\section{Detection method of omentin-1 level}

All the subjects were fasted for over 12 hours to collect $2 \mathrm{~mL}$ of fasting venous blood. After adding $120 \mu \mathrm{L}$ of aprotinin, the blood sample was centrifuged at 3,000 r/min to separate the plasma. And the level of omentin-1 in plasma was detected by the enzyme-linked immunosorbent method (ELISA).

\section{The content and methods}

Well-trained professional doctors collected the clinic data of this survey questionnaire, and the source of clinic data collection was based primarily on the patient's clinical medical history. Data were collected, including gender, age, tumor location, TNM stage, differentiation, pathological type, tumor type, lymph node metastasis, the situation of CRC patients.

\section{Standards for recurrence and metastasis after follow-up}

Postoperative follow-up was conducted by telephone, 
Table 1 Comparison of general conditions and plasma omentin-1 levels between the colorectal cancer group and the control group $(\bar{x}+\mathrm{S})$

\begin{tabular}{lccc}
\hline \multirow{2}{*}{ Group } & \multirow{2}{*}{ Cases } & \multicolumn{2}{c}{ Omentin-1 $(\mathrm{ng} / \mathrm{mL})$} \\
\cline { 3 - 4 } & & Pre-operation & Post-operation \\
\hline Case group & 319 & $69.32 \pm 23.51$ & $52.81 \pm 20.26^{\star}$ \\
Control group & 300 & $37.85 \pm 15.37$ & $37.85 \pm 15.37$ \\
$t$ & & 19.581 & 10.302 \\
$\mathrm{P}$ & & $<0.001$ & $<0.001$ \\
\hline
\end{tabular}

*, compared with pre-operation, $\mathrm{P}<0.01$.

outpatient, and other ways. Follow-up was conducted every three months for the first year after the surgery; then, every six months for the next year. The follow-up time was up to March 31, 2020. Follow-up outcomes include recurrence and death. The recurrence and metastasis were defined by undergoing B-ultrasound, CT, X-ray, and other imaging examinations, and confirmed by clinical pathology. Then, the pathology was retrieved through colonoscopy and reoperation. The death case was retrieved through family members or retrieved relevant information from the Public Security Bureau.

\section{Statistical methods}

The measurement data is expressed as mean \pm standard deviation $(\bar{x}+\mathrm{s})$ and analyzed by the $t$-test of independent samples. The data within the group were compared using a paired $t$-test. Count data is expressed as a percentage number [n (\%)] and analyzed using the $\chi^{2}$ test. Independent risk factors of recurrence and survival were screened using multivariate COX regression analysis. The data was analyzed using SPSS 21.0 statistical software (IBM, USA), and results with $\mathrm{P}<0.05$ were considered statistically significant.

\section{Results}

\section{Comparison of plasma omentin-1 levels before and after surgery between the two groups}

The level of omentin-1 after surgery in the case group was significantly lower than that before surgery $(\mathrm{P}<0.05)$, while compared with the control group, the levels of omentin-1 before and after the surgery in the case group was significantly higher than the control group $(\mathrm{P}<0.05$, Table 1$)$.

\section{Comparison of plasma omentin-1 levels in patients with colorectal cancer with different pathological parameters}

Plasma omentin-1 levels were compared in colorectal cancer patients with different TNM stages and whether there were lymph node metastasis, and the difference was statistically significant $(\mathrm{P}<0.05)$. The plasma omentin-1 levels in patients with TNM stages III-IV and lymph node metastasis were significantly higher than patients with TNM stage I-II, no lymph node metastasis, and no vascular tumor thrombus (Table 2).

\section{Postoperative follow-up of CRC patient group}

All patients were followed up after surgery. During the follow-up period, four patients died within one month after surgery, and 24 patients and their family members could not be connected, so they were excluded from the failure of obtaining follow-up outcomes. Finally, 291 patients were enrolled. During the follow-up period, 89 patients had recurrence or metastasis, and the recurrence and metastasis rate was $30.58 \%$ (89/291). Among the 89 patients with recurrence or metastasis, 22 patients had local recurrence, 18 patients had a local recurrence with distant metastasis, and 49 patients had distant metastasis. During the whole follow-up period, 56 deaths occurred, with a mortality rate of $19.24 \%(56 / 291)$.

\section{Univariate analysis of recurrence and survival in patients with CRC}

The results of the univariate analysis showed that the recurrence rate of patients with TNM staging III-IV, poor differentiation, lymph node metastasis, vascular tumor thrombus, or omentin-1 level $\geq 50 \mathrm{ng} / \mathrm{mL}$ was significantly higher than that with TNM staging I-II, moderately and highly differentiated, no lymph node metastasis, no vascular tumor thrombus, omentin-1 level $<50 \mathrm{ng} / \mathrm{mL}$ $(\mathrm{P}<0.05)$. The 2 -year survival rate of the case group with poor differentiation, lymph node metastasis, vascular tumor thrombus, omentin-1 level $\geq 50 \mathrm{ng} / \mathrm{mL}$ was significantly lower than the group with moderately and highly differentiated, no lymph node metastasis, no vascular tumor thrombus, and omentin-1 level $<50 \mathrm{ng} / \mathrm{mL}(\mathrm{P}<0.05$, Table 3). 
Table 2 Comparison of plasma retinoin-1 levels of colorectal cancer patients with different pathological parameters $(\bar{x}+\mathrm{S}, \mathrm{ng} / \mathrm{mL})$

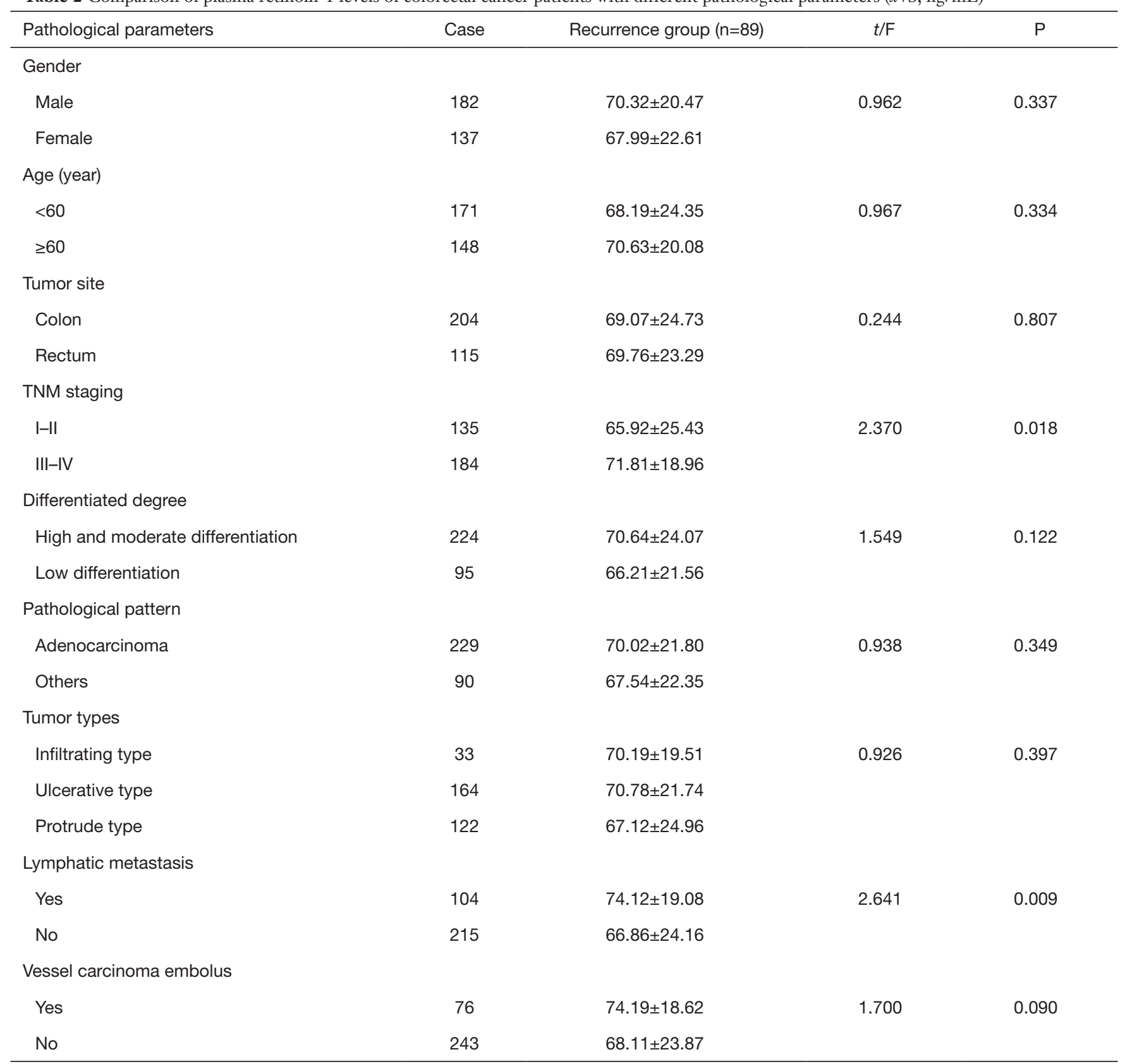

\section{Multivariate COX regression analysis of recurrence in patients with CRC}

Results of multivariate Cox regression analysis showed TNM staging III-IV, lymph node metastasis, omentin-1 level $\geq 50 \mathrm{ng} / \mathrm{mL}$ are independent risk factors for recurrence of CRC patients $(\mathrm{P}<0.05$, Table 4$)$.
Multivariate Cox regression analysis of multiple risk factors affecting the 2-year survival of patients with CRC

Results of multivariable Cox regression analysis showed that poor differentiation, lymph node metastasis, vascular tumor thrombus, omentin-1 level $\geq 50 \mathrm{ng} / \mathrm{mL}$ were independent risk factors affecting the 2-year survival of patients with 
Table 3 Single-factor analysis of recurrence and survival rate of colorectal cancer patients [n (\%)]

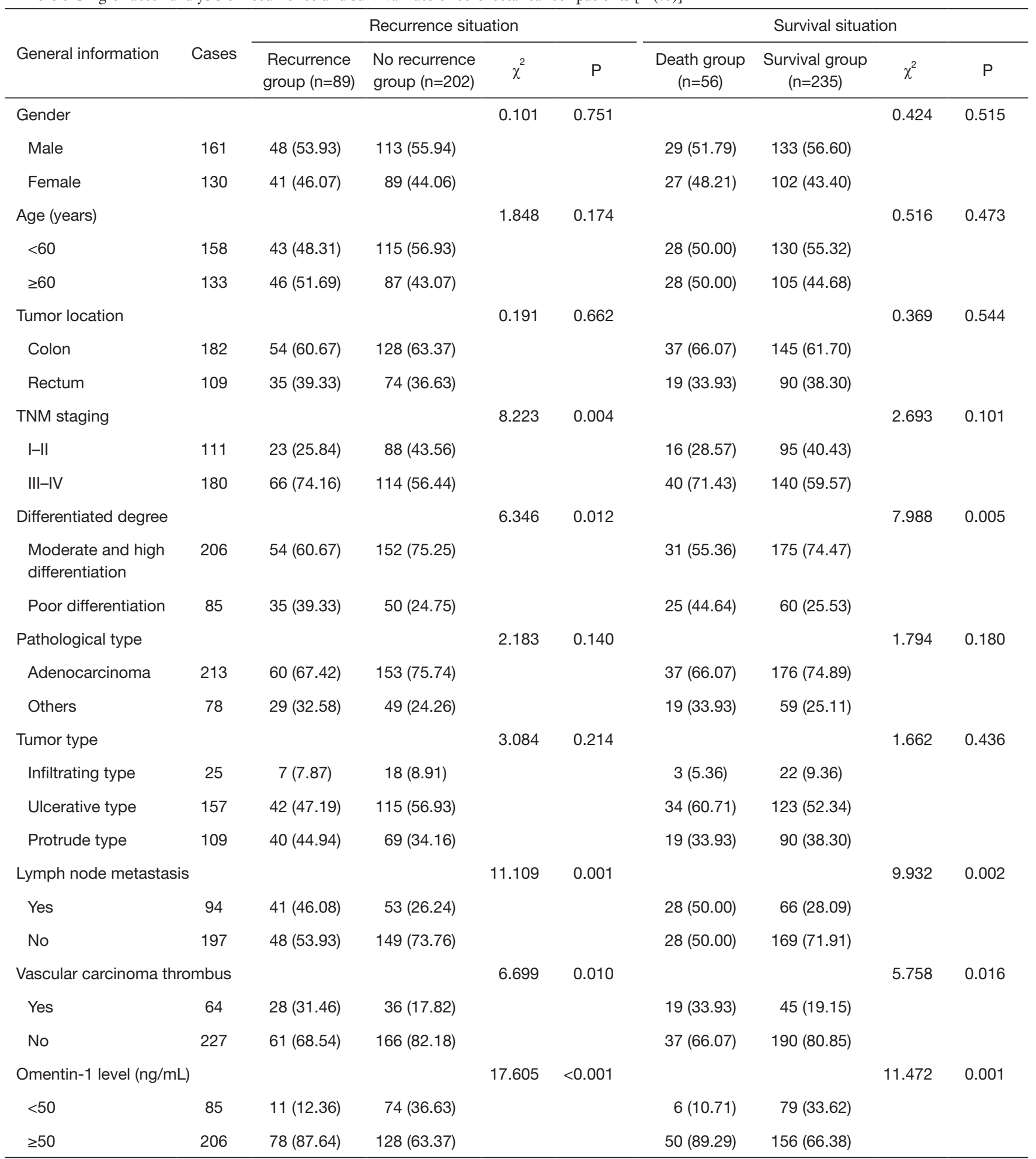


Table 4 Multivariate COX regression analysis of recurrence in patients with colorectal cancer

\begin{tabular}{lcccccc}
\hline Risk factors & $\beta$ & SE & wald $\chi^{2}$ & $\mathrm{P}$ & $\mathrm{OR}$ & $95 \% \mathrm{Cl}$ \\
\hline TNM staging of stage III-IV & 0.682 & 0.305 & 5.000 & 0.025 & 1.978 & $1.088-3.596$ \\
Poor differentiation & 0.579 & 0.317 & 3.336 & 0.068 & $0.959-3.321$ \\
With lymph node metastasis & 0.729 & 0.288 & 6.407 & 0.011 & 2.073 & $1.179-3.645$ \\
With vascular carcinoma thrombus & 0.647 & 0.346 & 3.497 & 0.061 & 1.910 & $0.969-3.763$ \\
Omentin-1 level $\geq 50 \mathrm{ng} / \mathrm{mL}$ & 1.208 & 0.439 & 7.572 & 0.006 & 3.347 & $1.416-7.912$ \\
\hline
\end{tabular}

Table 5 Multivariate COX regression analysis of factors affecting the 2-year survival of patients with colorectal cancer

\begin{tabular}{lcccccc}
\hline Risk factors & $\beta$ & SE & wald $\chi^{2}$ & $\mathrm{P}$ & $\mathrm{OR}$ & $95 \% \mathrm{Cl}$ \\
\hline Poor differentiation & 0.673 & 0.351 & 3.676 & 0.055 & 1.960 & $0.985-3.900$ \\
With lymph node metastasis & 0.897 & 0.359 & 6.243 & 0.012 & 2.452 & $1.213-4.956$ \\
With vascular carcinoma thrombus & 0.706 & 0.342 & 4.261 & 0.039 & 2.026 & $1.036-3.960$ \\
Omentin-1 level $\geq 50 \mathrm{ng} / \mathrm{mL}$ & 1.185 & 0.496 & 5.708 & 0.017 & 3.271 & $1.237-8.647$ \\
\hline
\end{tabular}

CRC $(\mathrm{P}<0.05$, Table 5).

\section{Discussion}

Colorectal cancer, as most common human malignant tumor with the third morbidity and fifth death rate, has received more and more attention from clinicians and experts and scholars. The risk factors that affect the development and prognosis of colorectal cancer are also increasingly refined to some microRNAs, phosphatase-tensin gene and other microscopic molecules $(10,11)$, including some endocrine adipokines. Surgical resection is the primary clinical treatment for patients with CRC, which shows a significant short-term effect, but the high recurrence and metastasis rate in the later stages still seriously threatens the safety of patients (9). How to find the relapse of patients as early as possible and then perform early intervention treatment is the key point to prolong the life of patients with CRC. The research on the risk factors affecting the prognosis of CRC patients has gradually transferred from the macroscopic angle, including age, TNM staging, and lymph node metastasis, to the microscopic molecular perspectives, including microRNA and genes including phosphatase tensin (PTEN) $(10,11)$.

The relationship between endocrine adipokines and CRC has also received increased attention. Some researchers believe that adipokines may be related to the occurrence and development of CRC (5). It has been shown adiponectin, a type of adipocytokine, is a risk factor for the onset of CRC (12). Other important adipocytokines, omentin-1, can inhibit the proliferation and promote the apoptosis of colon cancer stem cells via the PI3K/Akt signaling pathway (13), which is closely related to the patient's condition.

The results of this study showed that the level of plasma omentin-1 in the CRC case group after surgery was significantly decreased, but still higher than that of the control group. And among patients with colorectal cancer, the level of plasma omentin-1 of pateints with TNM staging III-IV and lymph node metastasis was significantly higher than that of TNM staging I-II or without lymph node metastasis $(\mathrm{P}<0.05)$. Omentin-1 is a significant factor secreted by adipocytes, selectively expressed in omental adipose tissue and highly expressed in plasma. Studies have shown that omentin- 1 has excellent sensitivity and specificity for distinguishing patients with and without CRC. A sensitivity of over $80 \%$ and a specificity of around $70 \%$ was observed (14). Meanwhile, the significant reduction of the omentin-1 level after surgery shows omentin-1 is related to the condition of patients with CRC. Research also shows omentin-1 can promote the proliferation of colon cancer cell lines SW480 (15), and can also inhibit its apoptosis simultaneously, which shows omentin-1 might be used as a biomarker for the development of CRC patients.

Follow-up results in this study showed that the recurrence and metastasis rate was $30.58 \%(89 / 291)$, and the mortality rate was $19.24 \%$ (56/291), which shows better efficacy 
than the previously reported recurrence rate of $60 \%$ (1) and metastasis rate of $41.35 \%$ (3). This phenomenon may receive help from the current development of medical technology. The results of the multivariable analysis showed TNM staging, lymph node metastasis, and how much differentiation is all independent risk factors affecting the recurrence rate and survival of patients, which are consistent with the results of other studies $(1,16)$. Patients with higher TNM stage and poor differentiation of tumors have a higher malignant, the tumor, and a higher probability of metastasis, which directly affects the patient's postoperative recurrence and survival time (17). As one of the significant ways of tumor metastasis, the metastasis rate of lymph node metastasis has a significant relation to the long-term prognosis of patients $(18,19)$. However, because of the different conditions of patients and different followup times, recurrence and mortality are inevitably different, and the influencing factors and results are also different. Besides, results in this study also showed that omentin-1 was an independent risk factor affecting the recurrence and metastasis of CRC patients, and an independent risk factor affecting patient survival. It is believed in some literature that omentin-1 can activate the intracellular serine/ threonine-protein kinase Akt in the absence of insulin by enhancing the phosphorylation of Akt, which can promote cell proliferation and metabolism, inhibit cell apoptosis, and increase the secretion of angiogenic cytokines to affect the permeability and regeneration of blood vessels and play an essential role in carcinogenicity (5). The study of mouse bone marrow mesenchymal hepatocyte experiment shows that that omentin-1 can promote the secretion of vascular growth factors by vascular endothelial cells and improve the ability of stem cells to induce angiogenesis. While after blocking PI3K/Akt signaling, the effect of omentin-1 on the proliferation of stem cells disappeared (20). Therefore, during the development of CRC, omentin-1 plays a role in promoting the proliferation of tumor cells and the formation of peripheral blood vessels by activating the Akt related signaling pathways. Simultaneously, it can also increase the permeability of blood vessels to be more conducive to the metastasis of tumor cells, shortening the survival time of patients.

In summary, plasma omentin-1 levels are highly expressed in patients with CRC, and is closely related to TNM staging and lymph node metastasis, which is an independent factor affecting postoperative recurrence and survival, suggesting that plasma omentin-1 might be a biomarker to predict the recurrence of colorectal patients.

\section{Acknowledgments}

Funding: None.

\section{Footnote}

Reporting Checklist: The authors have completed the REMARK reporting checklist. Available at http://dx.doi. org/10.21037/tcr-20-2836

Data Sharing Statement: Available at http://dx.doi. org/10.21037/tcr-20-2836

Conflicts of Interest: All authors have completed the ICMJE uniform disclosure form (available at http://dx.doi. org/10.21037/tcr-20-2836). The authors have no conflicts of interest to declare.

Ethical Statement: The authors are accountable for all aspects of the work in ensuring that questions related to the accuracy or integrity of any part of the work are appropriately investigated and resolved. This study was approved by Beijing Luhe Hospital, Affiliated to Capital Medical University (No. 2020-LHKY-033-01). The study was conducted in accordance with the Declaration of Helsinki (as revised in 2013). Written informed consent was obtained from the patient.

Open Access Statement: This is an Open Access article distributed in accordance with the Creative Commons Attribution-NonCommercial-NoDerivs 4.0 International License (CC BY-NC-ND 4.0), which permits the noncommercial replication and distribution of the article with the strict proviso that no changes or edits are made and the original work is properly cited (including links to both the formal publication through the relevant DOI and the license). See: https://creativecommons.org/licenses/by-nc-nd/4.0/.

\section{References}

1. Dekker E, Tanis PJ, Vleugels JLA, et al. Colorectal cancer. Lancet 2019;394:1467-80.

2. Mattiuzzi C, Sanchis-Gomar F, Lippi G. Concise update on colorectal cancer epidemiology. Ann Transl Med 2019;7:609.

3. Tsutsui M, Yamamoto S. Impact of hypochloremia in resected colorectal cancer patients. Chin Clin Oncol 2019;8:S16.

4. Wu G, Li J, Qin C. Reduced RANBP9 expression is 
associated with poor prognosis in colorectal cancer patients. Transl Cancer Res 2019;8:2704-12.

5. Pothuraju R, Rachagani S, Junker WM, et al. Pancreatic cancer associated with obesity and diabetes: an alternative approach for its targeting. J Exp Clin Cancer Res 2018;37:319.

6. Yang J, Zaman MM, Vlasakov I, et al. Adipocytes promote ovarian cancer chemoresistance. Sci Rep 2019;9:13316.

7. Uyar GO, Sanlier N. Association of adipokines and insulin, which have a role in obesity, with colorectal cancer. Eurasian J Med 2019;51:191-5.

8. Ji H, Wan L, Zhang Q, et al. The effect of omentin-1 on the proliferation and apoptosis of colon cancer stem cells and the potential mechanism. J BUON 2019;24:91-8.

9. De Rosa M, Pace U, Rega D, et al. Genetics, diagnosis and management of colorectal cancer (Review). Oncol Rep 2015;34:1087-96.

10. Asano H, Kojima K, Ogino N, et al.. Postoperative recurrence and risk factors of colorectal cancer perforation. Int J Colorectal Dis 2017;32:419-24.

11. Fletcher R, Wang YJ, Schoen RE, et al. Colorectal cancer prevention: Immune modulation taking the stage. Biochim Biophys Acta Rev Cancer 2018;1869:138-48.

12. Otani $\mathrm{K}$, Ishihara $\mathrm{S}$, Yamaguchi $\mathrm{H}$, et al. Adiponectin and colorectal cancer. Surg Today 2017;47:151-8.

13. Ji H, Wan L, Zhang Q, et al. The effect of omentin-1 on the proliferation and apoptosis of colon cancer stem cells and the potential mechanism. J BUON 2019;24:91-8.

14. Zhao X, Zhang Y, Deng L, et al. The association between Chinese patients'elevated omentin-1 levels, their clinicopathological features, and the risk of colorectal cancer. Int J Clin Exp Pathol 2019;12:2264-74.

15. Ying J, Tsujii $M$, Kondo J, et al. The effectiveness of an anti-human IL-6 receptor monoclonal antibody combined with chemotherapy to target colon cancer stem-like cells. Int J Oncol 2015;46:1551-9.

16. Wei Q, Huang X, Fu B, et al. IMP3 expression in biopsy specimens of colorectal cancer predicts lymph node metastasis and TNM stage. Int J Clin Exp Pathol 2015;8:11024-32.

17. Anitei MG, Zeitoun G, Mlecnik B, et al. Prognostic and predictive values of the immunoscore in patients with rectal cancer. Clin Cancer Res 2014;20:1891-9.

18. Tjan-Heijnen V, Viale G. The Lymph Node and the Metastasis. N Engl J Med 2018;378:2045-6.

19. Jin M, Frankel WL. Lymph node metastasis in colorectal cancer. Surg Oncol Clin N Am 2018;27:401-12.

20. Yin L, Huang D, Liu X, et al. Omentin-1 Effects on mesenchymal stem cells: proliferation, apoptosis, and angiogenesis in vitro. Stem Cell Res Ther 2017;8:224.

(English Language Editor: J. Chapnick)

Cite this article as: Feng Z, Sun H, Liu P, Shi W, Han W, $\mathrm{Ma} \mathrm{L}$. Analysis of the expression of plasma omentin-1 level in colorectal cancer and its correlation with prognosis. Transl Cancer Res 2020;9(10):6479-6486. doi: 10.21037/tcr-20-2836 